\title{
A Mathematical Approach to Merging Data from Different Trace Gas/Particulate Sensors Having Dissimilar (T90) Response Times: Application to Fire Emission Factor Determination
}

\author{
Tianran Zhang ${ }^{1,2 *}$, Martin Wooster ${ }^{1,2}$, David C. Green ${ }^{3}$, Bruce Main ${ }^{1}$ \\ ${ }^{1}$ Department of Geography, King's College London, Strand, London, WC2R 2LS, UK \\ ${ }^{2}$ National Centre for Earth Observation (NCEO), King's College London, Strand, London, WC2R 2LS, UK \\ ${ }^{3}$ Environmental Research Group, MRC-PHE Centre for Environment and Health, King's College London, London, \\ SE1 9NH, UK
}

\begin{abstract}
Low cost atmospheric composition sensors are increasingly used in both air quality research and in air pollution monitoring. Those focused on particulates have generally rather rapid (T90) response times due to their reliance on light scattering methods. However, those electrochemical and NDIR sensors targeting trace gases such as, respectively, $\mathrm{CO}$ and $\mathrm{CO}_{2}$ typically respond very much slower, with $\mathrm{T} 90$ response times that can be further lengthened by the rate at which sample exchange occurs in the measurement cell. Data from these types of low cost sensors are often used to derive emission ratios [ERs] of two simultaneously measured atmospheric species, yet ER derivations made using data from sensors having dissimilar T90 values can be problematic, because any rapid change in the pollutant concentration can mean one sensor responding faster to the changing conditions than the other. Such situations are typical within biomass burning plumes, yet where such ER assessments are routinely required to generate the emissions factors (EFs) needed for biomass burning emissions calculations. Here we confirm that the ERs coming from such analyses can be strongly biased if differential sensor T90 effects remain unadjusted for, and we demonstrate a simple mathematical approach for undertaking this adjustment. The method is able to take data from a sensor taken with a particular T90 value and simulate that which would be collected from a sensor targeting the same species but with a different T90 value, and if the output T90 is selected to match that of the companion sensor measuring the second species then the two measures with the same effective T90 values can be used to generate unbiased emissions ratios and emissions factors. We demonstrate our approach on simulated data with known T90 response times, and then apply it to real data from low-cost sensors collected within biomass burning plumes, including those installed in a sampling system that introduced further - and unknown additional measurement lag. In the latter case we find that our mathematical approach to the post-measurement T90 adjustment is still able to derive ERs from the low-cost sensor data that are comparable to those derived from data collected with a very high precision laser absorption spectrometer that measures the two species exactly simultaneously. Our methodology is thus well suited to the problem of deriving emissions ratios and emission factors from data collected by low-cost sensing systems deployed in rapidly changing pollutant plumes.
\end{abstract}

Keywords: Low-cost sensor; Carbon dioxide; Carbon monoxide; Biomass burning.

\section{INTRODUCTION}

Low cost trace gas sensors are increasingly used in both industry and environmental science for assessing the concentrations of target species. Such measurements typically use either electrochemical (e.g., for $\mathrm{CO}, \mathrm{SO}_{2}, \mathrm{HS}, \mathrm{NO}_{2}$; Zampolli et al., 2004; Mead et al., 2013; Piedrahita et al., 2014) or non-dispersive infrared (NDIR) methods (e.g., for

\footnotetext{
${ }^{*}$ Corresponding author.

E-mail address: tianran.zhang@kcl.ac.uk
}

$\mathrm{CO}_{2}$; Yasuda et al., 2012; Piedrahita et al., 2014), but in environments characterised by rapidly changing atmospheric concentrations there can be problems introduced when comparing data from different sensors having dissimilar sensor response times. Environments where this issue occurs in extreme ways include volcanic plumes (Roberts et al., 2012) and biomass burning plumes (Zhang et al., 2015) for example, and here the differential response times of the two sensors can greatly bias any derived emission ratio [ER] between the two measured species (Roberts et al., 2012). Since biomass burning trace gas emission factors (EFs) are commonly derived from such ER measures (e.g., Zhang et al., 2015), the derived EFs can also suffer from strong 
biases. Similar biases can occur when deriving particulate emissions factors if the data collected by the different particle and trace gas sensors are not adjusted for the differing sensor response times (e.g., as demonstrated by Wooster et al., 2018).

Roberts et al. (2012) detail how small, low-cost electrochemical and NDIR trace gas sensors are typically characterised by a significant 'lag' in their response to changing species concentrations. Most respond exponentially when exposed to a changed trace gas abundance, and this characteristic is generally specified via a T90 metric, representing the time after which the sensor is outputting a measured concentration that is the equivalent of $90 \%$ of the true concentration it has been newly exposed to. For a sensor sampled at e.g., $1 \mathrm{~Hz}$, the output concentration $\left[\mathrm{I}_{\text {Sensor }}(\mathrm{t}), \mathrm{ppmv}\right]$ measured at time $[\mathrm{t}, \mathrm{secs}]$ is a function of the gas mixing ratio $[\mathrm{X}(\mathrm{t}), \mathrm{ppmv}]$ and the sensor sensitivity $\left(\mathrm{S}_{\text {Sensor }}\right.$, unitless). This $\mathrm{I}_{\text {Sensor }}(\mathrm{t})$ is ascertained from the final reading obtained after an exposure time significantly longer than the quoted T90 response time (i.e., typically for many minutes), and depends on the 'slowness factor' [F, unitless], the sensor output at the previous timestep [ $\mathrm{I}_{\text {Sensor }}(\mathrm{t}-1), \mathrm{ppmv}$ ], and a sensor baseline [ $\left.\mathrm{I}_{\text {SensorBaseline }}(\mathrm{t}), \mathrm{ppmv}\right]$ which is generally pre-set in the lab to be near-zero for the types of electrochemical and NDIR sensors being considered here.

$I_{\text {Sensor }}(t)=\left[X_{t}\right] \cdot S_{\text {Sensor }} \cdot(1-F)+I_{\text {Sensor }}(t-1) \cdot F+$

$I_{\text {SensorBaseline }}(t)$

The slowness factor $(F)$ is normally directly inferable from the quoted T90 value, which can normally be obtained from the relevant technical documentation for the sensor, or which can be assessed from laboratory measurements of gas samples of known concentrations (Roberts et al., 2012, 2014):

$$
F=e^{\frac{\operatorname{Ln}(0.1)}{T_{90}}}
$$

However, a problem arises when sensors having different T90 values are exposed to rapidly changing concentrations, yet their data need to be compared. This occurs for example in pollutant plumes, in conditions where wind speed and direction change rapidly, or where portable personal monitoring sensors are moved rapidly from one environment to another. In such circumstances concentrations of the target species can change on timescales faster than the sensor T90 values, meaning that any derived emission ratio extracted from comparisons of the data from the two sensors can show significant bias (Reid and Hobbs., 1998; Zhang et al., 2015).

Fig. 1 provides an example of a $\mathrm{CO}$ measurement made in-situ in a biomass burning plume, similar to that described in Wooster et al., (2018). The volumetric mixing ratio of $\mathrm{CO}$ is measured by two sensors in this example, a highprecision, cavity enhanced laser absorption spectrometer supplied by Los Gatos Research (LGR) with a T90 of less than 6 seconds (governed by its internal gas cell volume and rate of flow; CO LGR, Table 1), and a small, low cost electrochemical $\mathrm{CO}^{-}$sensor $\left(\mathrm{CO}_{\mathrm{AX}}\right)$ with a far longer T90 of 40 seconds (this being related primarily to the diffusion of gas onto the sensor surfaces and the speed of the chemical reactions involved. Further details of these two different measurement systems are provided in Section 3, but from Fig. 1(a) it is clear that whilst the CO_LGR timeseries shows three very clear discrete plumes (peaks of 200, 100 and 75 ppmv of CO respectively), the output from the CO AX sensor essentially combines the first and second of these plumes due to its far longer sensor response time. As a result, when plotting the two $\mathrm{CO}$ mixing ratios against one another (Fig. 1(b)) a hysteresis effect is apparent, directly
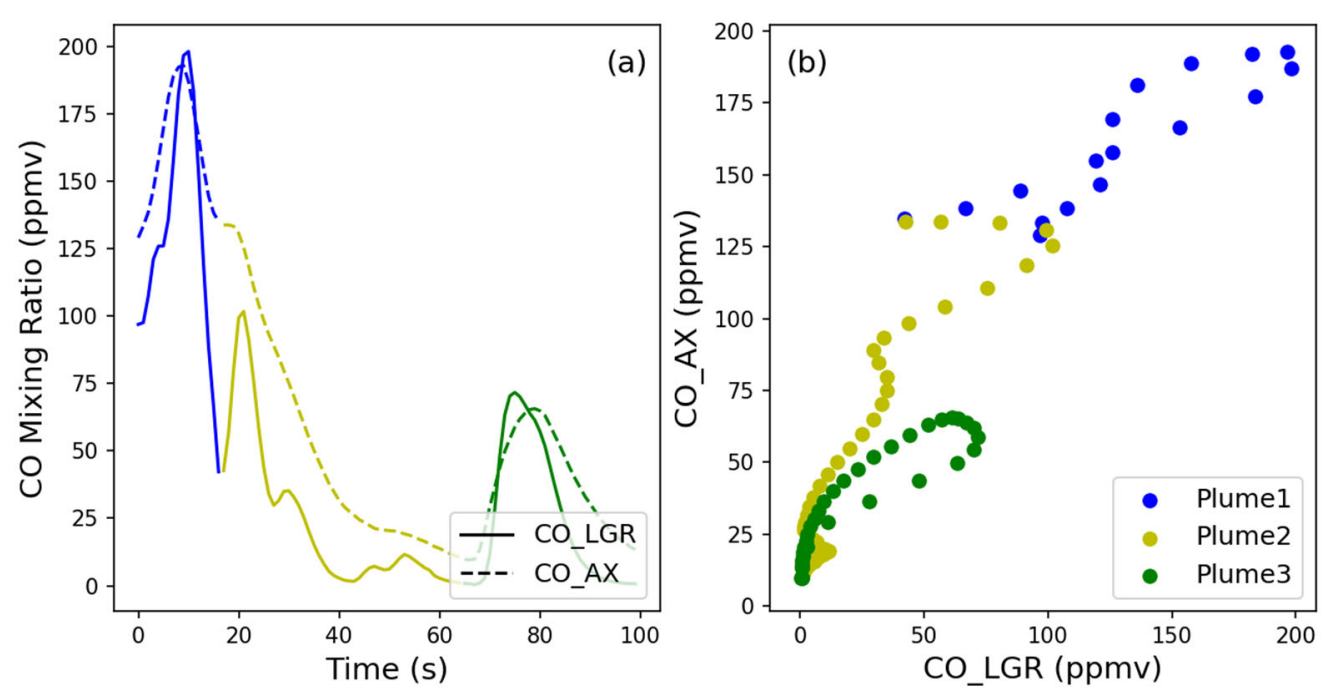

Fig. 1. Simultaneous carbon monoxide (CO) measurements made in a biomass burning plume by two different sensors, a fast responding LGR laser absorption spectrometer (CO_LGR), and a far slower responding electrochemical sensor (CO_AX). (a) Timeseries from the two sensors in which three plumes (coloured differently) can be picked out in the LGR timeseries, but only two of which are separable in the CO_AX timeseries. (b) Scatterplot of the trace gas concentrations measured by the two sensors, with the hysteresis caused by the differing sensor response times. 
Table 1. Fuel and sensors used herein.

\begin{tabular}{lll}
\hline Fire & Fuel type & Sensors Used \\
\hline 1 & Wheat Crop Post-Harvest Residue (China) & LGR, NDIR \\
2 & Straw (UK) & AQ, LGR \\
3 & Tropical Peat (Indonesia) & Dusttrak \\
\hline
\end{tabular}

related to the differing sensor response times. Clearly if the LGR sensor in the Fig. 1 example was measuring a trace gas other than $\mathrm{CO}$ (e.g., $\mathrm{CO}_{2}$ or $\mathrm{CH}_{4}$, which it can also measure), then the emissions ratios derived in the standard way from a linear best fit to the two trace gas concentrations (e.g., Wooster et al., 2018) could be negatively impacted. Reducing the potential for this unwanted effect is the aim of this work, and whilst we focus specifically on the derivation of biomass burning emissions ratios the approach we demonstrate is equally appropriate for deployment in other rapidly changing pollutant situations.

\section{TWO TRADITIONAL METHODS: TEMPORAL SHIFT AND DATA AVERAGING}

Many biomass burning emissions studies have compared measurements of multiple species within the same plume, made using different sensors. To overcome the impact of any difference in the T90 response times, some have applied simple time shifts to the temporally coincident measurements to make them appear to align more effectively (e.g., Chen et al., 2007; Hörtnagl et al., 2010). However, whilst such shifts can increase the linear correlation coefficient $(r)$ seen between the two trace gas concentrations, and thus make it appear that a more appropriate ER is derivable (with typically less uncertainty) than when using the original observations, Roberts et al. (2014) show that in fact the derived ERs can be significantly biased. This is because any applied temporal shift changes the gradient of the linear best fit away from that which characterises the true emission ratio, even if $r$ is increased in the process. Temporal averaging (or integrating) the two sets of data over fixed (e.g., 1-min) or variable (e.g., over the duration of each 'plume') time-periods is likely a far better approach to adjusting for the dissimilar T90 values of the sensors, and one that appears to induce far less ER biases (Roberts et $a l ., 2014)$. We compare the temporal shifting and temporal averaging approaches in Fig. 2, which shows simulated data representing the temporal sampling of a biomass burning plume. Several Gaussian distribution modes (calculated using Eq. (3)) are used to simulate various plumes sampled over time, each having random noise added to provide a realistic simulation of a real data record. Examples of such data are those from a sensor being transected back and forth through a plume, such as shown in e.g., Zhang et al. (2015) and Wooster et al. (2018) for biomass burning plumes in China's agricultural fires and Indonesian peat fires respectively.

$$
f(t)=\frac{A}{\sqrt{2 \pi \sigma^{2}}} e^{-\frac{(t-\mu)^{2}}{2 \sigma^{2}}}
$$

where $\mathrm{t}$ refers to the time and $\mu$ the mean time (secs) of each Gaussian mode, $\sigma$ is the standard deviation (assumed to be 1.0) and A refers to the total abundance of a mode/plume (on unitless scale of 10-100).

As shown in Fig. 2 and referred to here, 'Environmental' refers to the 'true' concentrations within the environment being sampled, whereas 'Detected' refers to the concentration reported by the simulated sampling system (as calculated in the simulation using Eqs. (1) and (2)). A unity gradient of the linear best fit between the 'Environmental' and 'Detected datasets would be the ideal case, and would be expected if the simulation of the "Detected" data was conducted with an assumed zero value T90. In this simulation however, a 40 -sec T90 was used (simulating that of the CO_AX sensor used by Zhang et al. (2015) to measure $\mathrm{CO}$ concentrations within biomass burning plumes), and consequently the Fig. 2(b) scatterplot shows a significant degree of scatter between the 'Environmental' and 'Detected' datasets - which in turn would introduce significant uncertainties into any derived emissions ratio. When a temporal shift is applied to the "Detected" data in an attempt to maximise the correlation coefficient $(r)$ between the two datasets (Fig. 2(c), 'Modified') the gradient of the resulting linear best fit is too high at 1.16 (i.e., if we were deriving emissions ratios, it would be $16 \%$ biased), but when the best fit it applied to unshifted $100 \mathrm{sec}$ temporal averages (Fig. 2(d)) the gradient becomes 1.0 and the correlation coefficient almost equal to unity. Hence, though the number of points (and the range of the absolute concentrations measurements) is lowered by the temporal averaging process shown in Fig. 2(d), the derived ER values are unbiased. Thus, according to this simulation, temporal averaging for periods far longer than the sensor T90 response times may allow unbiased trace gas emissions ratios to be derived from sensors measuring with dissimilar T90 values.

Zhang et al. (2015) already applied this temporal averaging method within a biomass burning plume study, using $20 \mathrm{sec}$ averaging intervals in the case where two trace gases were measured by sensors having different T90 response times, and up to 120 secs where concentrations of a single trace gas were ratioed against simultaneous aerosol measures made using an optical particle counter (which has effectively a close to zero T90 value). A disadvantage of the temporal averaging approach is, however, that it reduces the time resolution of the data, and also the amount of data available for emission ratio derivation (as can be seen when comparing Figs. 2(b) and 2(d)), and in the case of biomass burning studies this may lead to merging of data from different stages of the fire, for example the 'flaming' and 'smouldering' phases that sometimes occur at different times. This can prove challenging to cope with in cases 

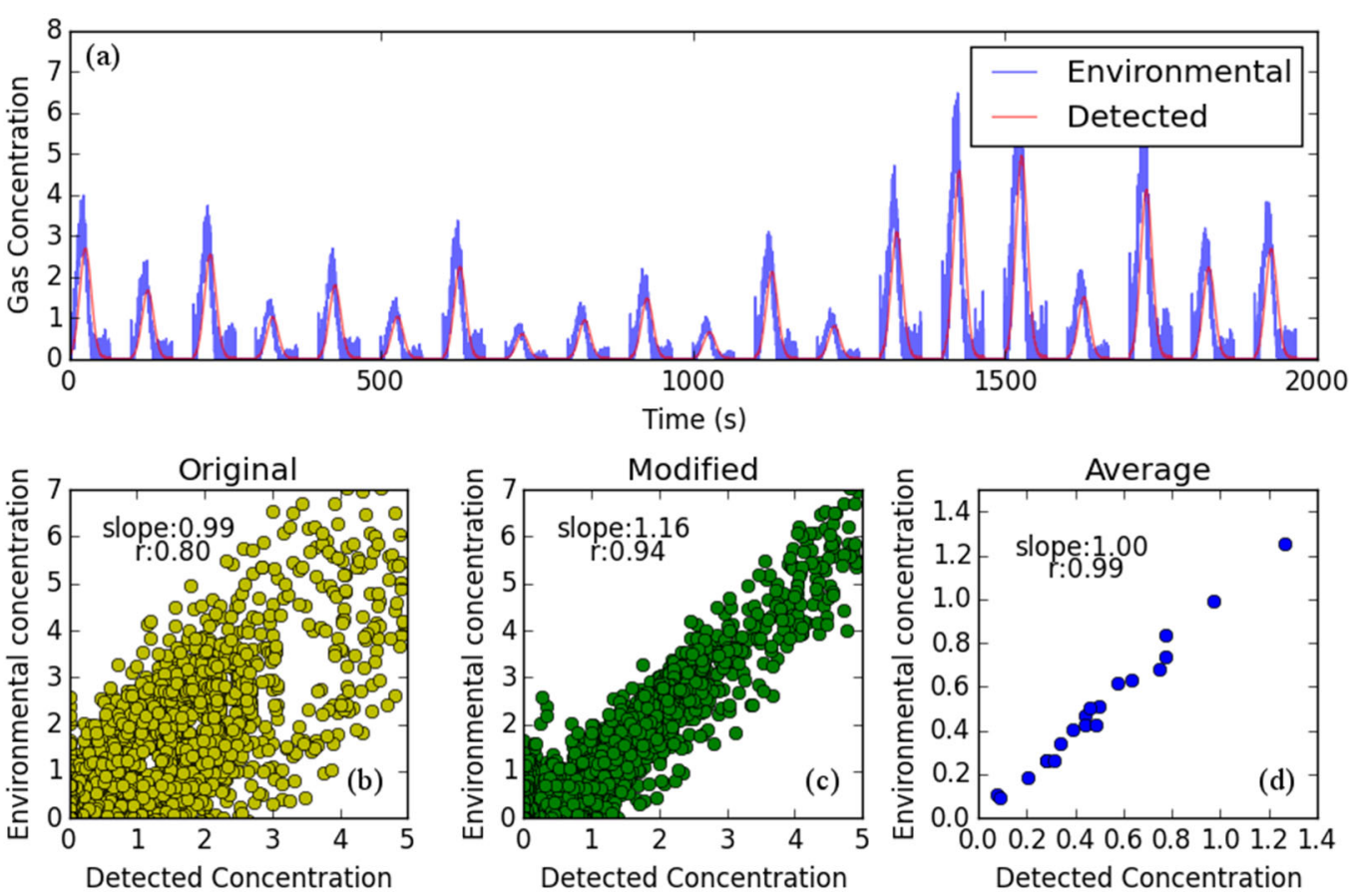

Fig. 2. Simulation of data where a biomass burning plume having true 'Environmental' concentrations of a particular trace gas is being sampled by an instrument having a $40 \mathrm{sec}$ T90. (a) Time series of the true 'Environmental' trace gas concentrations, along with the 'Detected' trace gas concentrations that represent the measured concentrations derived from the 'Environmental concentrations' via Eqs. (1) and (2) and an assumed T90 of 40 sec. Scatter plots between the 'Environmental' and 'Detected' concentrations, are shown with (b) the raw 'Detected' data used, along with that processed using (c) a temporal shift of 40 seconds, and (d) 100 second averaging.

where the measurement duration lasts only a few minutes (as can be the case in many field measurement situations), and in the simulation of Fig. 1 since each plume transect lasts only a few tens of seconds a temporal averaging window of 20 secs can certainly be envisaged to result in ERs that combine measurements made in different fire phases. There is therefore an interest in additional approaches which do not reduce the temporal resolution of the data when deriving unbiased ER values in such plumes, yet can cope with data from sensors with different response time values. Here we demonstrate such a method, based on a simple mathematical 'lag time adjustment' approach which we demonstrate on real biomass burning plume data taken under a variety of conditions.

\section{METHODOLOGY}

\section{Lag Time Adjustment Model}

Our approach is based on adaption of Eq. (1), where for gas $[X]$ the sensor measured value $[i(t)]$ at time $t$ is given by Eq. (4), assuming in Eq. 1 that $\mathrm{S}_{\text {Sensor }}$ is pre-set in the lab to be 1.0 to ensure that the digital output from each sensor responds $100 \%$ (albeit not instantaneously) to a change in the target gas concentration. $I_{\text {SensorBaseline }}$ is pre-set to zero since only excess abundances are used in our calculations.

$I_{i}(t)=[X] \cdot\left(1-F_{i}\right)+I_{i}(t-1) \cdot F_{i}$

Therefore, from the $I_{i}$ measurement time-series, the true gas concentration $[X]_{t}$ can be calculated as:

$$
[X]_{t}=\frac{I_{i}(t)-I_{i}(t-1) \cdot F_{i}}{\left(1-F_{i}\right)}
$$

and where $F_{i}$ (unitless) is taken from Eq. (2).

Eq. (5) then forms the basis of our method, and is aimed at deriving the true trace gas concentration timeseries from the measurements delivered by a sensor having a non-zero T90 value.

\section{Emission Factor and Emission Ratio Derivation}

Since one of the main reasons to collect trace gas and particulate concentration data in biomass burning plumes is for emission ratio and emissions factor derivation (Wooster et al., 2011; Zhang et al., 2015; Wooster et al., 2018), it is important that the concentrations derived via our Eq. (5) approach can be used to support this. EF determination in biomass burning plumes is generally based on the standard carbon mass balance method of Yokelson et al. (1999):

$$
E F_{x}=F_{c} \cdot 1000 \cdot \frac{M M_{x}}{M M_{c}} \cdot \frac{C_{x}}{C_{t}}
$$

where $F_{c}$ is the mass fraction of carbon in the fuel, $M M_{x}$ the molecular mass of the target gas, and $M M_{c}$ the molecular mass of carbon. 
Applications of the Eq. (6) approach to EF determination are included in detail in e.g., Yokelson et al. (1999), Goode et al. (1999), Sinha et al. (2003) and Wooster et al. (2011) for example. The ratio of the number of moles of species $x$ divided by the total number of moles of carbon $\left(C_{x} / C_{t}\right)$ is calculated according to Eq. (4), and relies on knowledge of the emission ratio, in this case of the trace gas $x$ in relation to the reference species $\mathrm{CO}_{2}\left[E R_{x / C O 2}\right]$ :

$$
\frac{C_{x}}{C_{t}}=\frac{E R_{x} / C O_{2}}{\sum_{j=1}^{n}\left(N C_{j} E R_{\frac{j}{C O_{2}}}\right)}
$$

This ER is generally derived from the gradient of the linear best fit to the measured excess abundances of species $[x]$ and the reference species $[y]$ (Yokelson et al.; 1999), though it is possible to also use the absolute rather than excess concentrations (Wooster et al., 2011).

\section{Test Data Collection}

We tested our approach using data collected from a variety of biomass burning situations and a variety of fuel types (Table 1), with the various sensors used listed in Table 2. Prior field research (e.g., Oanh et al., 2011) has suggested that in higher winds combustion can become more efficient, which may lead to different emission factors than in lower wind conditions, and so we ensured that we used data from relatively stable and calm wind conditions $\left(<3 \mathrm{~m} \mathrm{~s}^{-1}\right)$ to avoid changes in EF over time resulting from such an impact.

\section{RESULTS}

\section{Lag Time Model Simulation for $\mathrm{CO}_{2}$ Sensors}

Here we use data from two different $\mathrm{CO}_{2}$ sensors, a lowcost NDIR and a highly accurate laser absorption spectrometer [LGR]; see Table 2) measuring in the same biomass burning plume. Data were recorded on Fire 1 (Table 1) to evaluate the efficacy of our lag time adjustment approach, in the same way as for the simulated data detailed in Section 1. Since both $\mathrm{CO}_{2}$ sensors are measuring the same trace gas species, the 'emissions ratio' derived from the linear best fit to their data should be unity. During

Table 2. Sensors, their target species and estimated T90 response time.

\begin{tabular}{lll}
\hline Sensor & Species & T90 (secs) \\
\hline NDIR & $\mathrm{CO}_{2}$ & 20 \\
CO2_LGR & $\mathrm{CO}_{2}$ & $\sim 1-6^{*}$ \\
AQ & $\mathrm{CO}_{2}$ & $>40$ \\
CO_LGR & $\mathrm{CO}$ & $\sim 1-6^{*}$ \\
CO_AX & $\mathrm{CO}$ & 40 \\
AQ_ & $\mathrm{CO}$ & $>40$ \\
Dusttrak & PM2.5 & $\sim 1$ \\
\hline
\end{tabular}

* depends on whether using internal or external pump. When using an external pump with larger flow, the lag time can reduce to $\sim 1 \mathrm{~s}$. this test, three T90 values were assumed; 0 to represent instantaneous response, 3 secs to represent the T90 of the LGR sensor, and 20 secs to represent the T90 of the NDIR sensor. Fig. 3(a) shows the original unadjusted data, to which our lag time adjustment approach (Eq. (5)) was applied assuming each of these T90 response times in Eq. (2). Figs. 3(b), 3(c) and 3(d) show the resulting datasets and derived ERs.

Fig. 3(a) shows clearly that the $\mathrm{CO}_{2}$ data time series recorded with the NDIR sensor is smoother than that from the faster-responding LGR sensor. During the entire 300 sec sampling period, three main peaks can be found in the NDIR time-series. These are present in the LGR data, but there are also a number of additional (albeit smaller) peaks (particularly after 150 secs) largely absent in the NDIR data. A key difference occurs between 50 and 100 secs, where the LGR data but not the NDIR data shows a bimodal peak. Clearly the NDIR data show a greater lag time effect, as a result of the sensors longer T90 response time (Table 2).

Figs. 3(b) and 3(c) show the result of our lag time adjustment approach applied to the data of Fig. 3(a) using an assumed T90 value of 0 secs and 3 secs respectively. Though certainly not perfect, the output 'post-processed' NDIR data appear much more similar to that of the 'postprocessed' LGR, particularly that calculated with the $3 \mathrm{sec}$ T90 (Fig. 3(c)) used to replicate the real T90 value of the LGR sensor. For example, the peak in the data seen around 50 to 100 secs now appears correctly bi-modal in the NDIR data, matching that from the LGR. With the $3 \mathrm{sec}$ T90 applied, the NDIR data also show the appearance of the smaller plumes that appear after 150 secs, but which were largely absent in the original NDIR record (Fig. 3(a)). However, the post-processed NDIR data does contain a significant amount of apparently random noise, and this is introduced by the method and most severely when a T90 of zero is assumed (Fig. 3(b)). Such noise can impact direct comparisons between the output data, and would in the case of data of two different trace gas species also introduce uncertainties into the derived emission ratios. A potentially better option is therefore to post-process the LGR data by applying the 'slow' $20 \mathrm{sec}$ T90 response time of the NDIR sensor, again using Eq. (2). The results of this approach are shown in Fig. 3(d), where the noise seen in Figs. 3(b) and $3(c)$ is completely absent, with a resulting time-series from both sensors rather similar to that of the original LGR data in both shape and magnitude. As seen in the right hand column of Fig. 3, the gradient of the linear best fit to these data post-processed with an assumed NDIR T90 $=20$ secs is $1.1(r=0.91)$. The gradient is not quite unity as would be ideal, and the non-zero intercept could be the result of the equipment having different responses to certain gas concentrations, or sampling from slightly different locations having dissimilar $\mathrm{CO}_{2}$ concentrations. However, the same gradients derived from the different calculations (Figs. 3(b)$3(d)$ ) prove that changing the slowness factor (F) does not impact the emission ratio retrieval, and these gradients are very different from the highly biased value of 0.8 derived from the unprocessed data record of Fig. 3(a). 

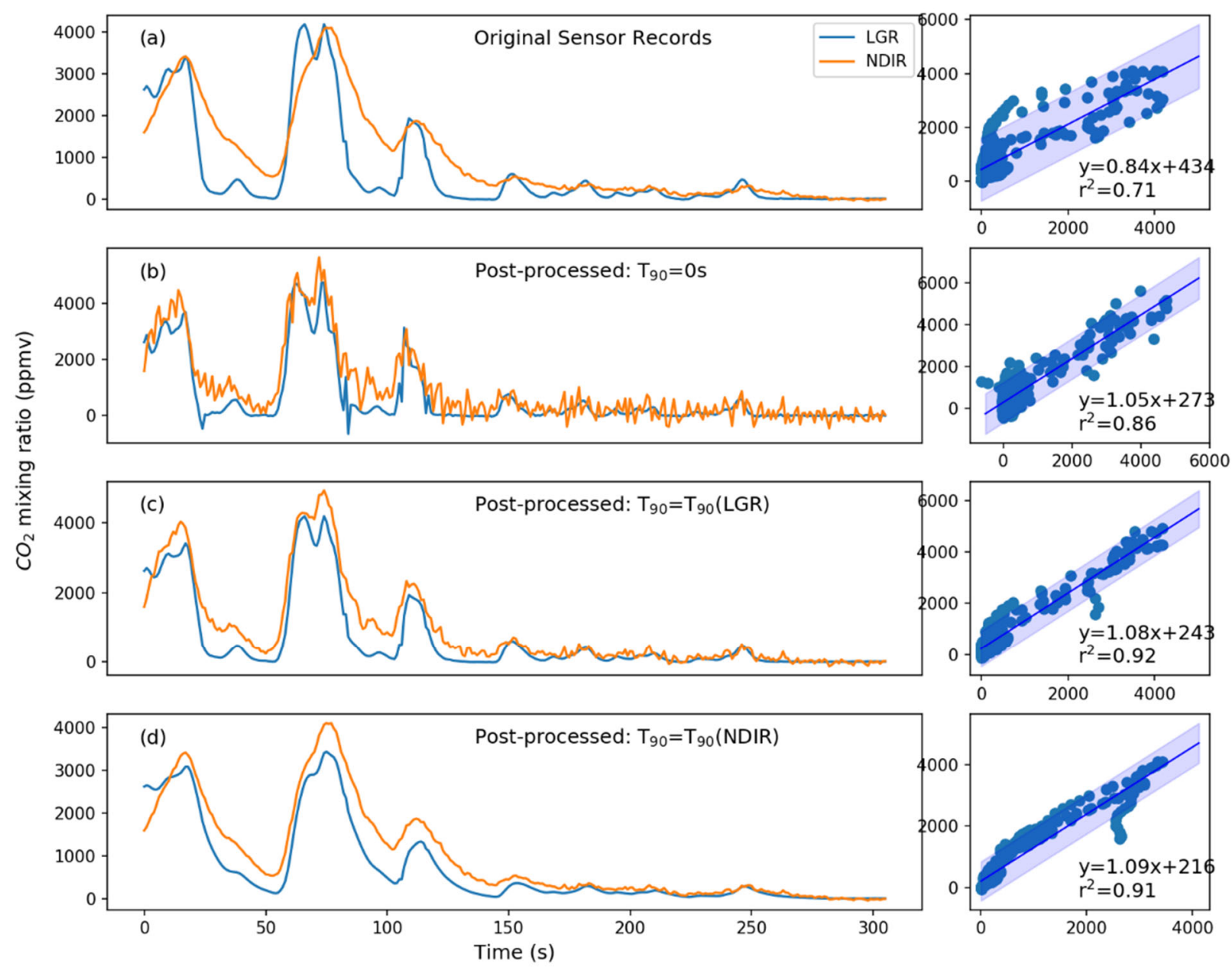

Fig. 3. Time series (left) and scatter plots (right) of $\mathrm{CO}_{2}$ mixing ratios as measured by the fast-response LGR laser absorption spectrometer and a slower response NDIR sensor. (a) original data, (b) NDIR data post processed with Eq. (7) but with an assumed T90 of 0 secs, (c) NDIR data post processed with Eq. (7) but with an assumed T90 of 3 secs (matching the response time of the LGR), and (d) LGR data post processed with Eq. (7) but with an assumed T90 of 20 secs (matching the NDIR sensor).

However, a further complication is that the typically quoted T90 for a sensor assumes it is directly deployed in the gas flow -whereas during real laboratory or in-situ measurements sensors are often encased in a more complex sampling system that may include tubing and chambers that increase further unknown 'slowness' into the response time (Mandayo et al., 2011; Zhang et al., 2015). We deal with this situation in the following Section.

\section{A Method to Derive Optimum Emission Ratios from Dual Trace Gas Data}

For sensors with unknown T90s, or which are fitted into sampling systems introducing additional 'slowness' (e.g., Zhang et al., 2015), a further optimised linear fitting approach is required. We demonstrate this using data from two sets of equipment, the first being the aforementioned LGR laser absorption spectrometer which can simultaneously measure $\mathrm{CO}$ and $\mathrm{CO}_{2}$ (Table 2) and with a near-zero response time when used with a fast external pump, and the second a commercial low cost air quality (AQ) meter incorporating a pair of low-cost sensors - electrochemical for
$\mathrm{CO}$ and NDIR for $\mathrm{CO}_{2}$ (Table 2) - that have unknown T90 response times. Four different smoke samples representing different smouldering and flaming combustion phases were fed into the two instruments, with the resulting $\mathrm{CO}_{2}$ and $\mathrm{CO}$ timeseries shown in Figs. 4(a) and 4(b) respectively. Whilst the LGR data show a rapid temporal response to the changing trace gas concentrations, the data from the AQ meter show a distinct lag, particularly with respect to $\mathrm{CO}_{2}$ (Fig. 4(b)). In the resulting scatterplots (Fig. 5), the LGR provides data from which it is directly possibly to derive strong linear best fits (Fig. 5(a)), whilst those from the AQ meter show distinct hysteresis effects of the kind associated with differential sensor response times (as discussed in Section 4.1).

To post-process the AQ data to remove the hysteresis and thus derive an unbiased and meaningful $\mathrm{CO}$ to $\mathrm{CO}_{2}$ emissions ratio, the data from the faster responding electrochemical $\mathrm{CO}$ sensor was subject to processing with Eqs. (2) and (5). The aim was to enable these data to be better compared to those from the slower responding $\mathrm{CO}_{2}$ sensor (i.e., that demonstrated in Fig. 5(b)). This processing 

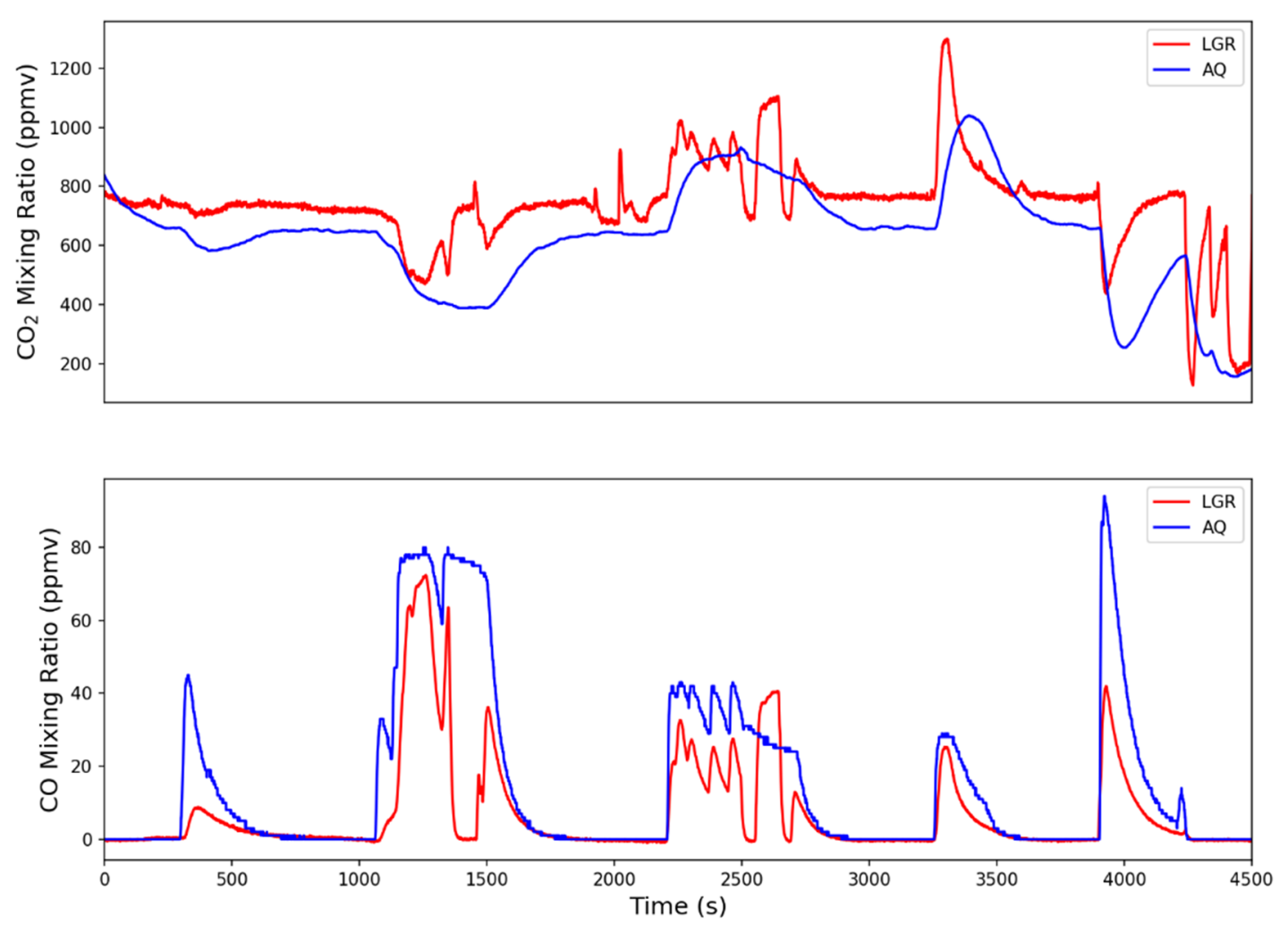

Fig. 4. $\mathrm{CO}_{2}$ and $\mathrm{CO}$ mixing ratio time series as measured by the fast-responding LGR and slower response NDIR and electrochemical air quality (AQ) sensors. The AQ meters $\mathrm{CO}_{2}$ sensor is significantly higher than that of the AQ CO sensor. Data is from Fire 2 (Table 1).
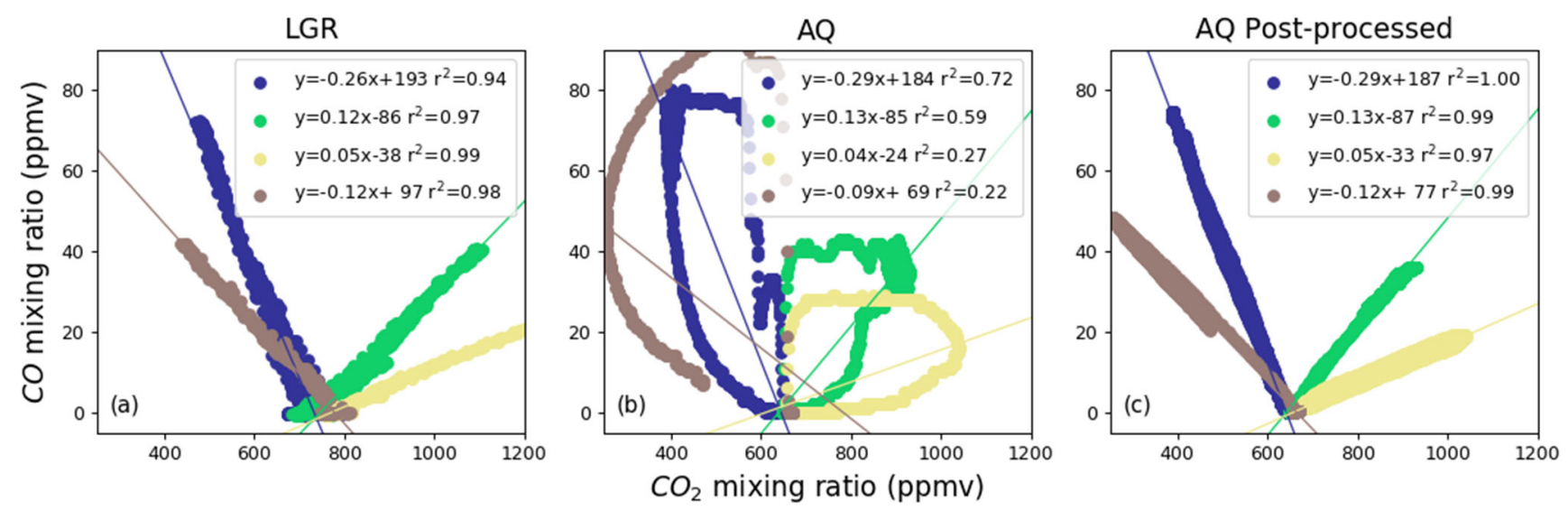

Fig. 5. Scatterplots of the $\mathrm{CO}$ to $\mathrm{CO}_{2}$ mixing ratios from the data shown of the four plumes of Fig. 4. (a) the LGR analyser, (b) original AQ sensor measurements, and (c) AQ sensors measurements post-processed to have the $\mathrm{CO}_{2} \mathrm{~T} 90$ equal to that of the slower responding $\mathrm{CO}_{2}$ sensor. Best fit linear relationships to the data from each plume are shown.

used a range of assumed T90 values within Eqs. (2) and (5), from 1 to 120 secs. The mean correlation coefficient $(\vec{r})$ for the $\mathrm{CO}$ and $\mathrm{CO}_{2}$ data of all plumes was used to select the lowest T90 providing an $\bar{r}$ exceeding 0.9 (selected to prevent overfitting). If none were found, then the T90 providing the highest $\bar{r}$ is selected. Subsequently, this postprocessed $\mathrm{CO}$ data was used with the un-processed (i.e., 'raw') $\mathrm{CO}_{2}$ data to derive the $\mathrm{CO}$ to $\mathrm{CO}_{2}$ emission ratio (Fig. 5(c)). Using this method, an optimised T90 of 74 secs was identified, which explains the serious lag shown in the
$\mathrm{CO}_{2}$ data recorded by the AQ instrument and shown in Fig. 4 compared to that from its companion $\mathrm{CO}$ sensor. The resulting emission ratios (as defined by the gradients of the linear best fit Equations) from the four smoke samples tested are $-0.29,0.13,0.05$ and -0.12 , values very close to those derived from the higher performing but far more costly and bulky LGR instrument $(-0.26,0.12,0.05,-0.12)$ which has a near-zero response time and measures all gases simultaneously. The percentage difference between the two sets of emissions ratios varies from 5 to $13 \%$, which 
compared to the gradients of the linear best fits to the 'raw' AQ data shown in Fig. 5(b) represents improvements of between 25 and $75 \%$.

\section{Derivation of Pollutant Plume Gas and Particle Data}

The method to derive optimised trace gas ERs described and demonstrated in Section 4.2 can also be used to derive particulate emissions ratios. ERs involving continuous or semi-continuous measurements of particulates and a reference trace gas underly field-based methods now often used for the derivation of biomass burning particulate matter (PM) emissions factors (e.g., Zhang et al. (2015) and Wooster et al. (2018)). Specifically, the PM emission factors can be derived from the emission ratio of the PM to the reference trace gas, normally $\mathrm{CO}$ or $\mathrm{CO}_{2}$, and the EF of that gas:

$E F_{P M}=a \cdot S_{P M / g a s} \cdot E F_{g a s}$

where $a$ is a unit conversion factor, with value of 0.909 ppm $\mathrm{mg}^{-1} \mathrm{~m}^{-3}$ when using $\mathrm{CO}_{2}$ as the reference gas, and $0.556 \mathrm{ppm} \mathrm{mg}^{-1} \mathrm{~m}^{-3}$ when using CO (Zhang et al., 2015; Wooster et al., 2018).

Here we demonstrate this method using the continuous $\mathrm{PM}_{2.5}$ measurements provided by a TSI Dusttrak laser photometer (Table 2), an instrument with an almost immediate response time (T90 $\cong 0)$ and which when appropriately calibrated using contemporaneous $\mathrm{PM}_{2.5}$

filter measurements can provide high quality data on $\mathrm{PM}_{2.5}$ abundance in biomass burning plumes (Wooster et al.,
2018). Fig. 6 shows an $\sim 30$ min time series of $\mathrm{CO}, \mathrm{CO}_{2}$ and $\mathrm{PM}_{2.5}$ collected during a biomass burning smoke sampling campaign (Fire 3, Table 1), with the dataset divided into five plumes for the analysis. $\mathrm{CO}_{2}$ and $\mathrm{CO}$ were measured using the aforementioned LGR laser absorption spectrometer (Table 2), with its internal pump giving an approximately 6 sec T90 for both species, whilst the $\mathrm{PM}_{2.5}$ measurements were collected by the Dusttrak.

When applying a linear best fit approach to the 'raw' LGR and Dusttrak data, very significant scatter is seen, with a linear best-fit correlation coefficient $(r)$ of 0.3 to 0.5 for each of the five plumes. After application of the optimisation method of Section 4.2, however, the post-processed Dusttrak data record now has a 'simulated' response time similar to that of the LGR, enabling a far higher correlation coefficient for the linear best fits (0.7-0.9) (Fig. 7(b)), which have gradients (i.e., PM to trace gas emissions ratios) changed by between 5 and $75 \%$ compared to those derived with the 'raw' data of Fig. 7(a). Most of these gradients have increase in value compared to those derived using the 'raw' data, as was the case in the simulation shown in Fig. 2.

Subsequent to the linear best fits shown in Fig. 7(b), the PM emissions factors can be derived from the particulate to trace gas ERs, and the results (Table 3) show that $\mathrm{EF}_{\mathrm{PM} 2.5}$ of all five plumes varies between 65 and $105 \mathrm{~g} \mathrm{~kg}^{-1}$ when using $\mathrm{CO}_{2}$ as the reference gas, and 73 to $111 \mathrm{~g} \mathrm{~kg}^{-1}$ when using $\mathrm{CO}$. The similarity of the values when using different gases as the reference further attests to the quality of the derived EF values.
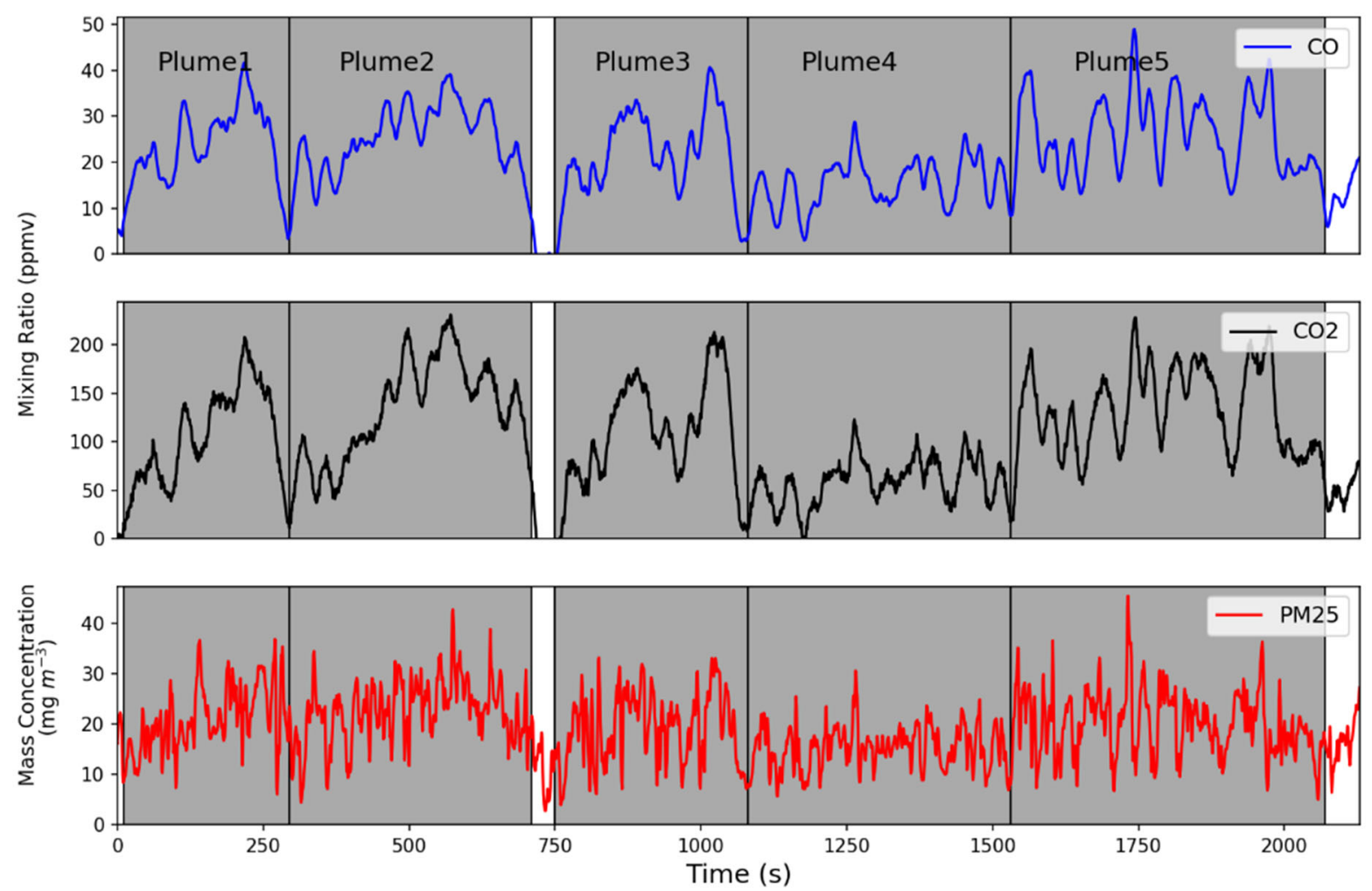

Fig. 6. Time series of $\mathrm{CO}$ and $\mathrm{CO}_{2}$ mixing ratios (ppmv), and simultaneously recorded $\mathrm{PM}_{2.5}$ concentrations (mg $\mathrm{m}^{-3}$ ) made during Fire 3 (Table 1). Data are segregated into that from five individual plumes. 

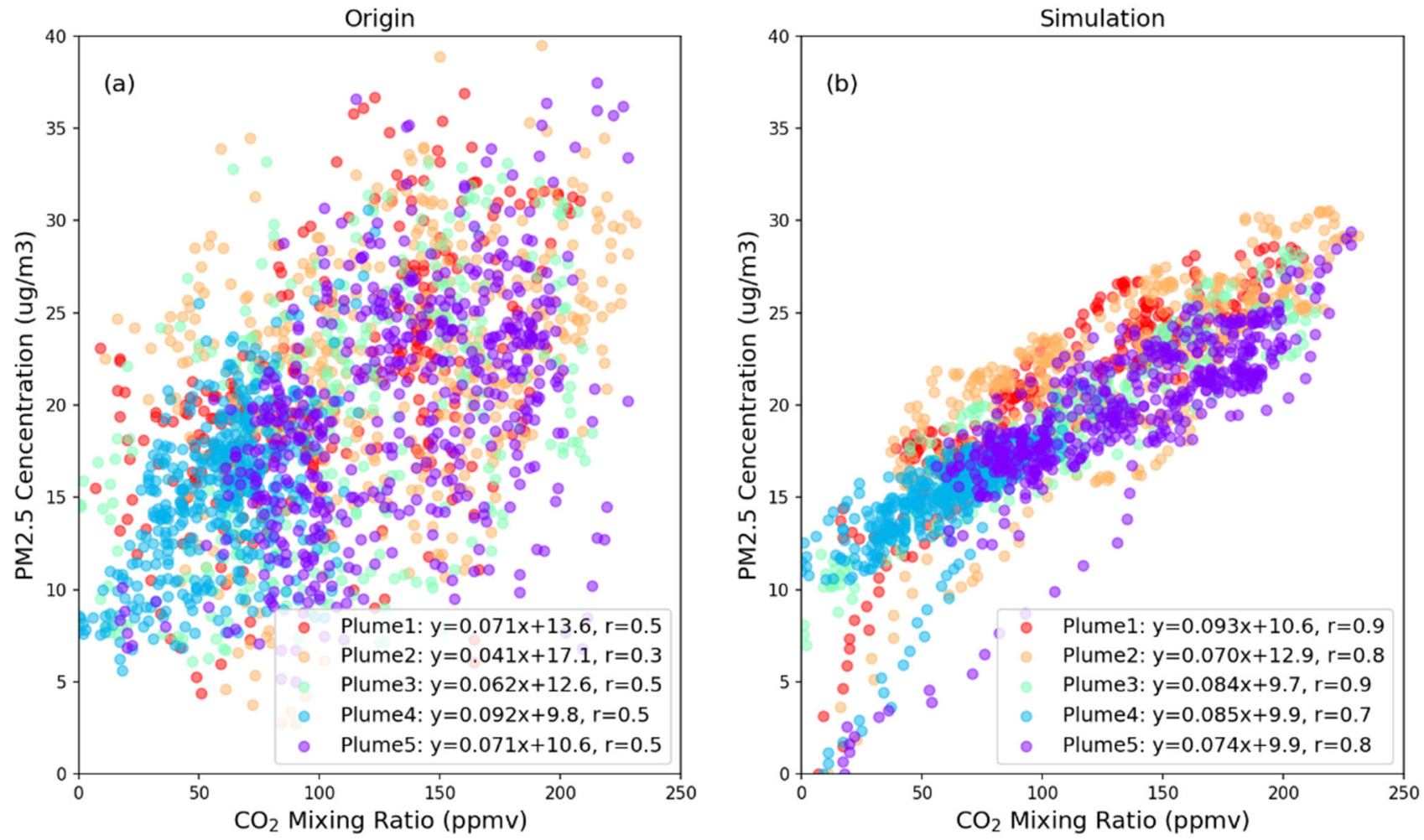

Fig. 7. Scatterplots of $\mathrm{PM}_{2.5}$ to $\mathrm{CO}$ taken from the measurements of Fire 2 (Table 1). The colours refer to those of the five different plumes shown in Fig. 6. (a) original data, and (b) data processed to have the $\mathrm{PM}_{2.5}$ time-series represent a T90 response time similar to that of the $\mathrm{CO}$ record.

Table 3. Emission factors (with uncertainties) of $\mathrm{CO}_{2}, \mathrm{CO}$, and $\mathrm{PM}_{2.5}$ derived separately from the ratio of particles to the two measured trace gases (i.e., data of Fig. 6) and the two-gas emission factors.

\begin{tabular}{lllll}
\hline & $\mathrm{EF} \mathrm{CO}_{2}$ & $\mathrm{EF} \mathrm{CO}$ & $\mathrm{EF} \mathrm{PM}_{2.5} \mathrm{CO}_{2}$ & $\mathrm{EF} \mathrm{PM}_{2.5} \mathrm{CO}$ \\
\hline Plume 1 & $1904 \pm 156$ & $194 \pm 16$ & $94 \pm 9$ & $98 \pm 9$ \\
Plume 2 & $1958 \pm 161$ & $164 \pm 14$ & $65 \pm 7$ & $73 \pm 7$ \\
Plume 3 & $1905 \pm 156$ & $194 \pm 16$ & $86 \pm 8$ & $93 \pm 9$ \\
Plume 4 & $1826 \pm 150$ & $241 \pm 20$ & $105 \pm 10$ & $111 \pm 10$ \\
Plume 5 & $1874 \pm 154$ & $213 \pm 18$ & $90 \pm 8$ & $96 \pm 9$ \\
\hline
\end{tabular}

\section{SUMMARY AND CONCLUSION}

Low cost, small electrochemical and NDIR sensors are increasingly used within scientific studies to assess changing trace gas concentrations, including within rapidly changing environments such as those associated with biomass burning plumes. Such sensors often demonstrate a significant lag in their response to the changing target species concentrations, characterised by non-zero T90 response times (the time taken for the sensor to record an output concentration $90 \%$ of that which it is being exposed to). These T90 values can be quite different between different sensors, and in some cases maybe unknown since they are influenced not only by the sensor itself but also by the plumbing and sample delivery system used to expose the sensors to the sampled plume. When measurement systems used to assess two trace gases have different T90 response times, any significant difference in the sensor T90 responses can introduce biases and/or uncertainty into any emissions ratio derived from the 'raw' observations of the two different species. However, we have demonstrated herein how use of a simple mathematical model proposed by Roberts et al. (2012) can enable post-processing of the 'raw' sensor data in a way that reduces or even removes the impact of differing T90 response times on any derived emissions ratio. We find that in order to minimise the amount of noise introduced into the post-processed data, the 'raw' output from the faster responding sensor should be processed to simulate the T90 of the slower responding sensor. Even where the T90 response time of the slower responding sensor is unknown we have demonstrated an optimisation method enabling unbiased ERs to be derived, for both trace gas and particulates. An alternative to the approach presented here is a simpler temporal averaging method whereby data from fixed interval periods ideally longer than the sensors T90 response time are averaged (or integrated) to provide the input data for a linear best-fit to be applied in order to generate the ER. This method is 
shown to give unbiased ERs, in contrast to a temporal shifting method that is demonstrated to introduce significant error, but does significantly lower the number of data points from which the ER is constructed. In the most serious case, plumes from different phases of combustion maybe averaged together by this processing procedure, for example leading to the combining of data from flamingand smouldering-dominated combustion and preventing the separate ERs from being explored. The approach provided here avoids this problem, but it is still recommended when collecting such data to bring the measurement sensors out of the pollutant stream every so-often to generate breaks in the measurement that can be used to isolate individual 'plumes' for further processing.

\section{ACKNOWLEDGEMENTS}

The authors would like to thank Dr. T. Roberts for helpful discussions during data analysis and based upon her published works. This work has been supported by funding from the Natural Environment Research Council (NERC) of the UK (PR140015 and NE/M017729/1) and by an EPSRC IAA Award (EP/R511559/1).

\section{REFERENCE}

Chen, S.J., Hovde, D.C., Peterson, K.A. and Marshall, A.W. (2007). Fire detection using smoke and gas sensors. Fire Saf. J. 42: 507-515.

Goode, J.G., Yokelson, R.J., Susott, R.A. and Ward, D.E. (1999). Trace gas emissions from laboratory biomass fires measured by open-path Fourier transform infrared spectroscopy: Fires in grass and surface fuels. $J$. Geophys. Res. 104: 21237-21245.

Hörtnagl, L., Clement, R., Graus, M., Hammerle, A., Hansel, A. and Wohlfahrt, G. (2010). Dealing with disjunct concentration measurements in eddy covariance applications: A comparison of available approaches. Atmos. Environ. 44: 2024-2032.

Mandayo, G.G., Herrán, J., Castro-Hurtado, I. and Castaño, E. (2011). Performance of a $\mathrm{CO}_{2}$ impedimetric sensor prototype for air quality monitoring. Sensors 11 : 5047-5057.

Mead, M.I., Popoola, O.A.M., Stewart, G.B., Landshoff, P., Calleja, M., Hayes, M., Baldovi, J.J., McLeod, M.W., Hodgson, T.F., Dicks, J. and Lewis, A. (2013). The use of electrochemical sensors for monitoring urban air quality in low-cost, high-density networks. Atmos. Environ. 7: 186-203.

Oanh, N.T.K., Ly, B.T., Tipayarom, D., Manandhar, B.R., Prapat, P., Simpson, C.D. and Liu, L.J.S. (2011). Characterization of particulate matter emission from open burning of rice straw. Atmos. Environ. 45: 493502.

Piedrahita, R., Xiang, Y., Masson, N., Ortega, J., Collier, A., Jiang, Y., Li, K., Dick, R.P., Lv, Q., Hannigan, M., and Shang, L. (2014). The next generation of low-cost personal air quality sensors for quantitative exposure monitoring, Atmos. Meas. Tech. 7: 3325-3336.
Reid, J.S. and Hobbs, P.V. (1998). Physical and optical properties of young smoke from individual biomass fires in Brazil. J. Geophys. Res. 103: 32013-32030.

Roberts, T.J., Braban, C.F., Oppenheimer, C., Martin, R.S., Freshwater, R.A., Dawson, D.H., Griffiths, P.T., Cox, R.A., Saffell, J.R. and Jones, R.L. (2012). Electrochemical sensing of volcanic gases. Chem. Geol. 332: 74-91.

Roberts, T.J., Saffell, J.R., Oppenheimer, C. and Lurton, T. (2014). Electrochemical sensors applied to pollution monitoring: Measurement error and gas ratio bias-a volcano plume case study. J. Volcanol. Geotherm. Res. . 281: 85-96.

Sinha, P., Hobbs, P.V., Yokelson, R.J., Bertschi, I.T., Blake, D.R., Simpson, I.J., Gao, S., Kirchstetter, T.W. and Novakov, T. (2003). Emissions of trace gases and particles from savanna fires in southern Africa. J. Geophys. Res. 108: 8487.

Wooster, M.J., Freeborn, P.H., Archibald, S., Oppenheimer, C., Roberts, G.J., Smith, T.E.L., Govender, N., Burton, M. and Palumbo, I. (2011). Field determination of biomass burning emission ratios and factors via openpath FTIR spectroscopy and fire radiative power assessment: headfire, backfire and residual smouldering combustion in African savannahs. Atmos. Chem. Phys. 11: 11591-11615.

Wooster, M.J., Gaveau, D.L.A., Salim, M.A., Zhang, T., $\mathrm{Xu}$, W., Green, D.C., Huijnen, V., Murdiyarso, D., Gunawan, D., Borchard, N., Schirrmann, M., Main, B. and Sepriando, A. (2018). New tropical peatland gas and particulate emissions factors indicate 2015 Indonesian fires released far more particulate matter (but less methane) than current inventories imply. Remote Sens. 10: 495.

Yasuda, T., Yonemura, S. and Tani, A. (2012) Comparison of the characteristics of small commercial NDIR $\mathrm{CO}_{2}$ sensor models and development of a portable $\mathrm{CO}_{2}$ measurement device. Sensors 12: 3641-3655.

Yokelson, R.J., Goode, J.G., Ward, D.E., Susott, R.A., Babbitt, R.E., Wade, D.D., Bertschi, I., Griffith, D.W. and Hao, W.M. (1999). Emissions of formaldehyde, acetic acid, methanol, and other trace gases from biomass fires in North Carolina measured by airborne Fourier transform infrared spectroscopy. J. Geophys. Res. 104: 30109-30125.

Zampolli, S., Elmi, I., Ahmed, F., Passini, M., Cardinali, G.C., Nicoletti, S. and Dori, L. (2004). An electronic nose based on solid state sensor arrays for low-cost indoor air quality monitoring applications. Sens. Actuators, B 101: 39-46.

Zhang, T., Wooster, M.J., Green, D.C. and Main, B. (2015). New field-based agricultural biomass burning trace gas, $\mathrm{PM}_{2.5}$, and black carbon emission ratios and factors measured in situ at crop residue fires in Eastern China. Atmos. Environ. 121: 22-34.

Received for review, February 1, 2019 Revised, June 17, 2019 Accepted, June 17, 2019 